\title{
Error Analysis for a Noisy Lacunary Cubic Spline Interpolation and a Simple Noisy Cubic Spline Quasi Interpolation
}

\author{
Feng-Gong Lang and Xiao-Ping Xu \\ School of Mathematical Sciences, Ocean University of China, Qingdao, Shandong 266100, China \\ Correspondence should be addressed to Feng-Gong Lang; fenggonglang@sina.com
}

Received 18 May 2014; Revised 30 August 2014; Accepted 30 August 2014; Published 10 September 2014

Academic Editor: Weizhu Bao

Copyright (C) 2014 F.-G. Lang and X.-P. Xu. This is an open access article distributed under the Creative Commons Attribution License, which permits unrestricted use, distribution, and reproduction in any medium, provided the original work is properly cited.

We mainly present the error analysis for two new cubic spline based methods; one is a lacunary interpolation method and the other is a very simple quasi interpolation method. The new methods are able to reconstruct a function and its first two derivatives from noisy function data. The explicit error bounds for the methods are given and proved. Numerical tests and comparisons are performed. Numerical results verify the efficiency of our methods.

\section{Introduction}

Cubic spline, as the most commonly used spline in practice, is a fundamental approximation tool [1-11]. Nowadays, it has been widely used in many fields such as numerical analysis, computer aided geometric design, mathematical modeling, and engineering problems. Essentially, cubic spline is a twice differentiable piecewise cubic polynomial defined over a partitioned interval.

Mathematically, cubic spline interpolation is often introduced as follows. Let $y(x)$ be a function defined over $[a, b]$, let

$$
y_{j}=y\left(x_{j}\right), \quad j=0,1, \ldots, n,
$$

be a set of given function data at the nodes

$$
a=x_{0}<x_{1}<\cdots<x_{n-1}<x_{n}=b,
$$

and let

$$
y_{0}^{\prime}=y^{\prime}\left(x_{0}\right), \quad y_{n}^{\prime}=y^{\prime}\left(x_{n}\right)
$$

be two boundary derivatives. Then, there exists a unique cubic spline $s(x)$ satisfying

$$
\begin{aligned}
& s^{\prime}\left(x_{0}\right)=y_{0}^{\prime}, \quad s^{\prime}\left(x_{n}\right)=y_{n}^{\prime}, \\
& s\left(x_{j}\right)=y_{j}, \quad j=0,1, \ldots, n .
\end{aligned}
$$

However, we often meet two troubles in the practical applications of cubic spline interpolation. The first trouble is that we cannot obtain the precise function values in (1). They generally involve some unavoidable measurement noise. The second trouble is that it often lacks the boundary derivatives in (3).

To deal with the troubles, in this paper, we give two new effective cubic spline based methods for reconstructing $y(x)$, $y^{\prime}(x)$, and $y^{\prime \prime}(x)$ from the given noisy data

$$
\tilde{y}_{j}=y\left(x_{j}\right)+\varepsilon_{j}, \quad j=0,1, \ldots, n,
$$

where $\varepsilon_{j}$ is the measurement noise. The first one is a noisy lacunary interpolation method (Method $I$ ) and the second one is a very simple noisy quasi interpolation method (Method II). The error bounds of the methods, which have not been studied before and are important and useful for the users of cubic spline, are mainly studied in this paper.

We organize the remainder of this paper as follows. In Section 2, we present some useful preliminaries; in Section 3, we give the new methods; in Section 4, we present the theoretical results of the errors; in Section 5, we perform some numerical tests to verify the error analysis; finally, we conclude this paper in Section 6. 


\section{Preliminaries}

2.1. Cubic B-Splines. We assume that the nodes in (2) are equidistant because this case is very common in practice. The nodes produce a uniform partition $\Delta$ for $[a, b]$ with mesh size $h=(b-a) / n$. The dimension of the cubic spline space over $\Delta$ is $n+3$. The corresponding cubic $\mathrm{B}$-splines are given below [4-8]. For $i=2,3, \ldots, n-2$, let

$$
B_{i}(x)=\frac{1}{6 h^{3}} \begin{cases}\left(x-x_{i-2}\right)^{3}, & \text { if } x \in\left[x_{i-2}, x_{i-1}\right] \\ \left(x-x_{i-2}\right)^{3}-4\left(x-x_{i-1}\right)^{3}, & \text { if } x \in\left[x_{i-1}, x_{i}\right] \\ \left(x_{i+2}-x\right)^{3}-4\left(x_{i+1}-x\right)^{3}, & \text { if } x \in\left[x_{i}, x_{i+1}\right] \\ \left(x_{i+2}-x\right)^{3}, & \text { if } x \in\left[x_{i+1}, x_{i+2}\right] \\ 0, & \text { else. }\end{cases}
$$

The other six B-splines $B_{-1}(x), B_{0}(x), B_{1}(x), B_{n-1}(x), B_{n}(x)$, and $B_{n+1}(x)$ are generated by the translation, where

$$
\begin{aligned}
& B_{-1}(x)=\frac{1}{6 h^{3}} \begin{cases}\left(x_{1}-x\right)^{3}, & \text { if } x \in\left[x_{0}, x_{1}\right] \\
0, & \text { else, }\end{cases} \\
& B_{0}(x)=\frac{1}{6 h^{3}} \begin{cases}\left(x_{2}-x\right)^{3}-4\left(x_{1}-x\right)^{3}, & \text { if } x \in\left[x_{0}, x_{1}\right] \\
\left(x_{2}-x\right)^{3}, & \text { if } x \in\left[x_{1}, x_{2}\right] \\
0, & \text { else, }\end{cases} \\
& B_{1}(x)=\frac{1}{6 h^{3}} \begin{cases}\left(x-x_{0}+h\right)^{3}-4\left(x-x_{0}\right)^{3}, & \text { if } x \in\left[x_{0}, x_{1}\right] \\
\left(x_{3}-x\right)^{3}-4\left(x_{2}-x\right)^{3}, & \text { if } x \in\left[x_{1}, x_{2}\right] \\
\left(x_{3}-x\right)^{3}, & \text { if } x \in\left[x_{2}, x_{3}\right] \\
0, & \text { else, }\end{cases} \\
& B_{n-1}(x)=\frac{1}{6 h^{3}} \begin{cases}\left(x-x_{n-3}\right)^{3}, & \text { if } x \in\left[x_{n-3}, x_{n-2}\right] \\
\left(x-x_{n-3}\right)^{3}-4\left(x-x_{n-2}\right)^{3}, & \text { if } x \in\left[x_{n-2}, x_{n-1}\right] \\
\left(x_{n}-x+h\right)^{3}-4\left(x_{n}-x\right)^{3}, & \text { if } x \in\left[x_{n-1}, x_{n}\right] \\
0, & \text { else, }\end{cases} \\
& B_{n}(x)=\frac{1}{6 h^{3}} \begin{cases}\left(x-x_{n-2}\right)^{3}, & \text { if } x \in\left[x_{n-2}, x_{n-1}\right] \\
\left(x-x_{n-2}\right)^{3}-4\left(x-x_{n-1}\right)^{3}, & \text { if } x \in\left[x_{n-1}, x_{n}\right] \\
0, & \text { else, }\end{cases} \\
& B_{n+1}(x)=\frac{1}{6 h^{3}} \begin{cases}\left(x-x_{n-1}\right)^{3}, & \text { if } x \in\left[x_{n-1}, x_{n}\right] \\
0, & \text { else. }\end{cases}
\end{aligned}
$$

They are linearly independent, nonnegative, and locally supported. Moreover,

$$
B_{i}^{(k)}(x)=B_{i+1}^{(k)}(x+h), \quad k=0,1,2 ; i=2,3, \ldots, n-3 .
$$

The values of $B_{i}(x), B_{i}^{\prime}(x)$, and $B_{i}^{\prime \prime}(x)$ at the nodes are listed in Table 1.
2.2. Approximate Boundary Derivatives. Using two-point numerical differentiation formula, we have

$$
\begin{aligned}
\tilde{y}_{0}^{\prime} & =\frac{\tilde{y}_{1}-\tilde{y}_{0}}{h}=\frac{y_{1}-y_{0}}{h}+\frac{\varepsilon_{1}-\varepsilon_{0}}{h} \\
& =y_{0}^{\prime}+\frac{h}{2} y^{\prime \prime}\left(\xi_{1}\right)+\frac{\varepsilon_{1}-\varepsilon_{0}}{h}=y_{0}^{\prime}+\varepsilon_{0}^{\prime}, \\
\tilde{y}_{n}^{\prime} & =\frac{\tilde{y}_{n}-\tilde{y}_{n-1}}{h}=\frac{y_{n}-y_{n-1}}{h}+\frac{\varepsilon_{n}-\varepsilon_{n-1}}{h} \\
& =y_{n}^{\prime}-\frac{h}{2} y^{\prime \prime}\left(\xi_{2}\right)+\frac{\varepsilon_{n}-\varepsilon_{n-1}}{h}=y_{n}^{\prime}+\varepsilon_{n}^{\prime} .
\end{aligned}
$$

Similar results can be obtained by using three-point and fivepoint numerical differentiation formulae. See Tables 2 and 3, where $\xi_{1} \in\left(x_{0}, x_{1}\right), \xi_{2} \in\left(x_{n-1}, x_{n}\right), \xi_{3} \in\left(x_{0}, x_{2}\right), \xi_{4} \in$ $\left(x_{n-2}, x_{n}\right), \xi_{5} \in\left(x_{0}, x_{4}\right)$, and $\xi_{6} \in\left(x_{n-4}, x_{n}\right)$ and $\varepsilon_{0}^{\prime}$ and $\varepsilon_{n}^{\prime}$ represent the computational truncated errors to $y^{\prime}\left(x_{0}\right)$ and $y^{\prime}\left(x_{n}\right)$. They arise from the used numerical differentiation formulae and the above-mentioned measurement noise.

\section{Two New Methods}

3.1. Method I. We study the following noisy lacunary cubic spline interpolation (NLCSI) problem. We hope to find a cubic spline $\widetilde{\mathcal{S}}(x)$ satisfying

$$
\widetilde{s}\left(x_{j}\right)=\widetilde{y}_{j}, \quad j=0,1,2, \ldots, n .
$$

To make the NLCSI problem uniquely solvable, it requires using two approximate boundary derivatives in Section 2.2. Obviously, there also exists a unique noisy lacunary cubic spline $\widetilde{s}(x)$ satisfying

$$
\begin{aligned}
& \widetilde{s}^{\prime}\left(x_{0}\right)=\tilde{y}_{0}^{\prime}, \quad \widetilde{s}^{\prime}\left(x_{n}\right)=\tilde{y}_{n}^{\prime}, \\
& \widetilde{s}\left(x_{j}\right)=\tilde{y}_{j}, \quad j=0,1,2, \ldots, n .
\end{aligned}
$$

Let $\widetilde{\boldsymbol{s}}(x)=\sum_{i=-1}^{n+1} \widetilde{c}_{i} B_{i}(x)$ be the cubic spline determined by (11), where the unknown coefficients $\widetilde{c}_{i}(i=-1,0, \ldots, n+1)$ can be obtained by solving the linear system

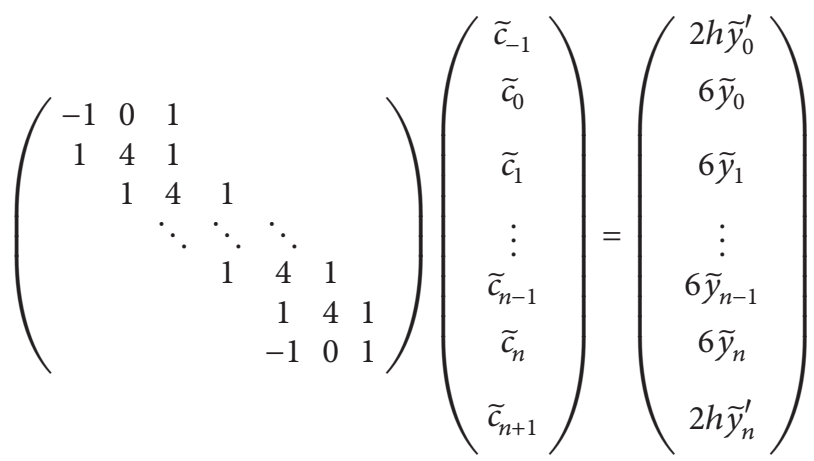

followed from Table 1. Furthermore, we can use $\vec{s}^{\prime}(x)=$ $\sum_{i=-1}^{n+1} \widetilde{c}_{i} B_{i}^{\prime}(x)$ and $\widetilde{s}^{\prime \prime}(x)=\sum_{i=-1}^{n+1} \widetilde{c}_{i} B_{i}^{\prime \prime}(x)$ to approximate $y^{\prime}(x)$ and $y^{\prime \prime}(x)$, respectively. 
TABLE 1: The values of $B_{i}^{(k)}(x)(i=-1,0, \ldots, n+1 ; k=0,1,2)$ at the nodes.

\begin{tabular}{ccccc}
\hline & $x_{i-1}$ & $x_{i}$ & $x_{i+1}$ & Else \\
\hline$B_{i}(x)$ & $\frac{1}{6}$ & $\frac{4}{6}$ & $\frac{1}{6}$ & 0 \\
$B_{i}^{\prime}(x)$ & $\frac{1}{2 h}$ & 0 & $-\frac{1}{2 h}$ & 0 \\
$B_{i}^{\prime \prime}(x)$ & $\frac{1}{h^{2}}$ & $-\frac{2}{h^{2}}$ & $\frac{1}{h^{2}}$ & 0 \\
\hline
\end{tabular}

TABLE 2: The approximate boundary derivatives and their errors (I).

\begin{tabular}{lcc}
\hline & Two-point results & Three-point results \\
\hline$\tilde{y}_{0}^{\prime}$ & $\frac{\tilde{y}_{1}-\tilde{y}_{0}}{h}$ & $\frac{-3 \widetilde{y}_{0}+4 \tilde{y}_{1}-\tilde{y}_{2}}{2 h}$ \\
\hline$\widetilde{\varepsilon}_{0}^{\prime}$ & $\frac{h}{2} y^{\prime \prime}\left(\xi_{1}\right)+\frac{\varepsilon_{1}-\varepsilon_{0}}{h}$ & $-\frac{h^{2}}{3} y^{\prime \prime \prime}\left(\xi_{3}\right)+\frac{-3 \varepsilon_{0}+4 \varepsilon_{1}-\varepsilon_{2}}{2 h}$ \\
\hline$\tilde{y}_{n}^{\prime}$ & $\frac{\tilde{y}_{n}-\tilde{y}_{n-1}}{h}$ & $\frac{\tilde{y}_{n-2}-4 \tilde{y}_{n-1}+3 \tilde{y}_{n}}{2 h}$ \\
\hline$\widetilde{\varepsilon}_{n}^{\prime}$ & $-\frac{h}{2} y^{\prime \prime}\left(\xi_{2}\right)+\frac{\varepsilon_{n}-\varepsilon_{n-1}}{h}$ & $-\frac{h^{2}}{3} y^{\prime \prime \prime}\left(\xi_{4}\right)+\frac{\varepsilon_{n-2}-4 \varepsilon_{n-1}+3 \varepsilon_{n}}{2 h}$ \\
\hline
\end{tabular}

TABLE 3: The approximate boundary derivatives and their errors (II).

\begin{tabular}{lc}
\hline & Five-point results \\
\hline$\widetilde{y}_{0}^{\prime}$ & $\frac{-25 \tilde{y}_{0}+48 \tilde{y}_{1}-36 \tilde{y}_{2}+16 \tilde{y}_{3}-3 \tilde{y}_{4}}{12 h}$ \\
\hline$\widetilde{\varepsilon}_{0}^{\prime}$ & $-\frac{h^{4}}{5} y^{(5)}\left(\xi_{5}\right)+\frac{-25 \varepsilon_{0}+48 \varepsilon_{1}-36 \varepsilon_{2}+16 \varepsilon_{3}-3 \varepsilon_{4}}{12 h}$ \\
\hline$\widetilde{y}_{n}^{\prime}$ & $-\frac{h^{4}}{5} y^{(5)}\left(\xi_{6}\right)+\frac{3 \varepsilon_{n-4}-16 \tilde{y}_{n-3}+36 \tilde{y}_{n-2}-48 \tilde{y}_{n-1}+25 \tilde{y}_{n}}{12 h}$ \\
\hline
\end{tabular}

3.2. Method II. By using the given function data, we can directly get a cubic spline

$$
\widetilde{\widetilde{s}}(x)=\sum_{i=-1}^{n+1} \tilde{y}_{i} B_{i}(x),
$$

where $\tilde{y}_{-1}=y\left(x_{0}-h\right)+\varepsilon_{-1}$ and $\tilde{y}_{n+1}=y\left(x_{n}+h\right)+\varepsilon_{n+1}$. We can also use $\tilde{\widetilde{s}}(x), \widetilde{\widetilde{s}}^{\prime}(x)$, and $\widetilde{\widetilde{s}}^{\prime \prime}(x)$ to approximate $y(x), y^{\prime}(x)$, and $y^{\prime \prime}(x)$, respectively.

The method is very simple and effective method for noisy data because it avoids using approximate boundary derivatives and also avoids solving the linear system (12).

\section{Main Results}

4.1. Error Analysis for Method I. We denote (12) by $A \widetilde{C}=\widetilde{Y}$.

Lemma 1. $A$ is invertible and $\left\|A^{-1}\right\|_{\infty} \leq 2$.
Proof. Add column one to column three and also add column $n+3$ to column $n+1$, and we get a strictly diagonally dominant matrix

$$
A_{1}=\left(\begin{array}{ccccccc}
-1 & 0 & 0 & & & & \\
1 & 4 & 2 & & & & \\
& 1 & 4 & 1 & & & \\
& & \ddots & \ddots & \ddots & & \\
& & & 1 & 4 & 1 & \\
& & & & 2 & 4 & 1 \\
& & & & 0 & 0 & 1
\end{array}\right)_{(n+3) \times(n+3)} .
$$

Obviously, $A_{1}$ is invertible and $A_{1}=A P$, where

$$
P=\left(\begin{array}{ccccccc}
1 & 0 & 1 & & & & \\
& 1 & 0 & & & & \\
& & 1 & & & & \\
& & & \ddots & & & \\
& & & & 1 & & \\
& & & & 0 & 1 & \\
& & & & 1 & 0 & 1
\end{array}\right)_{(n+3) \times(n+3)} .
$$

We have

$$
A^{-1}=P A_{1}^{-1}, \quad\left\|A_{1}^{-1}\right\|_{\infty} \leq 1, \quad\|P\|_{\infty}=2 .
$$

Hence, we have $\left\|A^{-1}\right\|_{\infty}=\left\|P A_{1}^{-1}\right\|_{\infty} \leq\|P\|_{\infty}\left\|A_{1}^{-1}\right\|_{\infty} \leq 2$.

Let $s(x)=\sum_{i=-1}^{n+1} c_{i} B_{i}(x)$ be the cubic spline determined by (4),

$$
\begin{aligned}
& C=\left(c_{-1}, c_{0}, \ldots, c_{n}, c_{n+1}\right)^{T}, \\
& Y=\left(2 h y_{0}^{\prime}, 6 y_{0}, \ldots, 6 y_{n}, 2 h y_{n}^{\prime}\right)^{T},
\end{aligned}
$$

and then we have $A C=Y$. Let

$$
\begin{aligned}
\varepsilon^{\prime} & =\max \left\{\left|\varepsilon_{0}^{\prime}\right|,\left|\varepsilon_{n}^{\prime}\right|\right\}, \\
\varepsilon & =\max _{0 \leq i \leq n}\left\{\left|\varepsilon_{i}\right|\right\},
\end{aligned}
$$

and we have

$$
\|\tilde{Y}-Y\|_{\infty} \leq \max \left\{2 h \varepsilon^{\prime}, 6 \varepsilon\right\}
$$

see Table 4 for the results, where $M_{5}=\left\|y^{(5)}(x)\right\|_{\infty}=$ $\max _{a \leq x \leq b}\left|y^{(5)}(x)\right|$ and $M_{4}, M_{3}$, and $M_{2}$ are defined similarly.

Lemma 2. Consider $\|\widetilde{C}-C\|_{\infty} \leq 2\|\widetilde{Y}-Y\|_{\infty}$.

Proof. Consider $\|\widetilde{C}-C\|_{\infty}=\left\|A^{-1}(\widetilde{Y}-Y)\right\|_{\infty} \leq$ $\left\|A^{-1}\right\|_{\infty}\|\widetilde{Y}-Y\|_{\infty} \leq 2\|\widetilde{Y}-Y\|_{\infty}$.

Lemma 3. Consider $\sum_{i=-1}^{n+1}\left|B_{i}(x)\right| \equiv 1, \sum_{i=-1}^{n+1}\left|B_{i}^{\prime}(x)\right| \leq 3 / 2 h$, and $\sum_{i=-1}^{n+1}\left|B_{i}^{\prime \prime}(x)\right| \leq 4 / h^{2}$. 
TABLE 4: The bounds of $\varepsilon^{\prime}$ and $\|\widetilde{Y}-Y\|_{\infty}$.

\begin{tabular}{lccc}
\hline & Two-point results & Three-point results & Five-point results \\
\hline$\varepsilon^{\prime} \leq$ & $\frac{1}{2} M_{2} h+\frac{2 \varepsilon}{h}$ & $\frac{1}{3} M_{3} h^{2}+\frac{4 \varepsilon}{h}$ & $\frac{1}{5} M_{5} h^{4}+\frac{32 \varepsilon}{3 h}$ \\
$\|\widetilde{Y}-Y\|_{\infty} \leq$ & $\max \left\{M_{2} h^{2}+4 \varepsilon, 6 \varepsilon\right\}$ & $\frac{2}{3} M_{3} h^{3}+8 \varepsilon$ & $\frac{2}{5} M_{5} h^{5}+\frac{64 \varepsilon}{3}$ \\
\hline
\end{tabular}

Proof. Because of property (8), we only need to check them over a typical subinterval $\left[x_{j}, x_{j+1}\right]$. By differentiating (6), for a general $i$, we have

$$
\begin{array}{r}
B_{i}^{\prime}(x)=\frac{1}{2 h^{3}} \begin{cases}\left(x-x_{i-2}\right)^{2}, & \text { if } x \in\left[x_{i-2}, x_{i-1}\right] \\
\left(x-x_{i-2}\right)^{2}-4\left(x-x_{i-1}\right)^{2}, & \text { if } x \in\left[x_{i-1}, x_{i}\right] \\
-\left(x_{i+2}-x\right)^{2}+4\left(x_{i+1}-x\right)^{2}, & \text { if } x \in\left[x_{i}, x_{i+1}\right] \\
-\left(x_{i+2}-x\right)^{2}, & \text { if } x \in\left[x_{i+1}, x_{i+2}\right] \\
0, & \text { else, }\end{cases} \\
B_{i}^{\prime \prime}(x)=\frac{1}{h^{3}} \begin{cases}x-x_{i-2}, & \text { if } x \in\left[x_{i-2}, x_{i-1}\right] \\
-3 x-x_{i-2}+4 x_{i-1}, & \text { if } x \in\left[x_{i-1}, x_{i}\right] \\
x_{i+2}-4 x_{i+1}+3 x, & \text { if } x \in\left[x_{i}, x_{i+1}\right] \\
x_{i+2}-x, & \text { if } x \in\left[x_{i+1}, x_{i+2}\right] \\
0, & \text { else. }\end{cases}
\end{array}
$$

All of them are locally supported over four adjacent subintervals.

(i) By the nonnegativity and partition of unity of cubic B-splines, for $x \in\left[x_{j}, x_{j+1}\right]$, we have

$$
\sum_{i=-1}^{n+1}\left|B_{i}(x)\right|=\sum_{i=-1}^{n+1} B_{i}(x)=\sum_{i=j-1}^{j+2} B_{i}(x) \equiv 1 .
$$

(ii) For $x \in\left[x_{j}, x_{j+1}\right]$, we have

$$
\begin{aligned}
& \sum_{i=-1}^{n+1}\left|B_{i}^{\prime}(x)\right| \\
& =\left|B_{j-1}^{\prime}(x)\right|+\left|B_{j}^{\prime}(x)\right|+\left|B_{j+1}^{\prime}(x)\right|+\left|B_{j+2}^{\prime}(x)\right| \\
& =\frac{1}{2 h^{3}}\left[\left(x-x_{j+1}\right)^{2}+\left|4\left(x-x_{j+1}\right)^{2}-\left(x-x_{j+2}\right)^{2}\right|\right. \\
& \left.\quad+\left|\left(x-x_{j-1}\right)^{2}-4\left(x-x_{j}\right)^{2}\right|+\left(x-x_{j}\right)^{2}\right] \\
& =\frac{1}{2 h^{3}}\left[-4\left(x-x_{j}\right)^{2}+4 h\left(x-x_{j}\right)+2 h^{2}\right] \leq \frac{3}{2 h} .
\end{aligned}
$$

(iii) For $x \in\left[x_{j}, x_{j+1}\right]$, we have

$$
\begin{aligned}
& \sum_{i=-1}^{n+1}\left|B_{i}^{\prime \prime}(x)\right| \\
& \quad=\left|B_{j-1}^{\prime \prime}(x)\right|+\left|B_{j}^{\prime \prime}(x)\right|+\left|B_{j+1}^{\prime \prime}(x)\right|+\left|B_{j+2}^{\prime \prime}(x)\right|
\end{aligned}
$$

$$
\begin{aligned}
& =\frac{1}{h^{3}}\left[\left(x_{j+1}-x\right)+\left|3 x+x_{j+2}-4 x_{j+1}\right|\right. \\
& \left.+\left|-3 x-x_{j-1}+4 x_{j}\right|+\left(x-x_{j}\right)\right] \\
& =\frac{1}{h^{3}} \begin{cases}4 h-6\left(x-x_{j}\right), & x \in\left[x_{j}, x_{j}+\frac{h}{3}\right] \\
2 h, & x \in\left[x_{j}+\frac{h}{3}, x_{j}+\frac{2 h}{3}\right] \\
6\left(x-x_{j}\right)-2 h, & x \in\left[x_{j}+\frac{2 h}{3}, x_{j+1}\right]\end{cases} \\
& \leq \frac{4}{h^{2}} \text {. }
\end{aligned}
$$

Lemma 4. Let $s(x)$ and $\widetilde{s}(x)$ be the cubic spline interpolants of $y(x)$ determined by (4) and (11), respectively. Then we have

$$
\begin{gathered}
|s(x)-\tilde{s}(x)| \leq 2\|\tilde{Y}-Y\|_{\infty}, \\
\left|s^{\prime}(x)-\widetilde{s}^{\prime}(x)\right| \leq \frac{3}{h}\|\tilde{Y}-Y\|_{\infty}, \\
\left|s^{\prime \prime}(x)-\widetilde{s}^{\prime \prime}(x)\right| \leq \frac{8}{h^{2}}\|\tilde{Y}-Y\|_{\infty} .
\end{gathered}
$$

Proof. First of all, for $k=0,1,2$, we have

$$
\begin{aligned}
\left|s^{(k)}(x)-\widetilde{s}^{(k)}(x)\right| & =\left|\sum_{i=-1}^{n+1}\left(c_{i}-\widetilde{c}_{i}\right) B_{i}^{(k)}(x)\right| \\
& \leq\|\widetilde{C}-C\|_{\infty} \sum_{i=-1}^{n+1}\left|B_{i}^{(k)}(x)\right| .
\end{aligned}
$$

And then using Lemmas 2 and 3, we get these results.

Theorem 5. Let $\widetilde{s}(x)$ be the noisy lacunary cubic spline interpolant of $y(x)$ determined by (11). Then one has

$$
\begin{aligned}
|\widetilde{s}(x)-y(x)| & \leq \frac{5}{384} M_{4} h^{4}+2\|\tilde{Y}-Y\|_{\infty}, \\
\left|\vec{s}^{\prime}(x)-y^{\prime}(x)\right| & \leq \frac{1}{24} M_{4} h^{3}+\frac{3}{h}\|\widetilde{Y}-Y\|_{\infty}, \\
\left|\widetilde{s}^{\prime \prime}(x)-y^{\prime \prime}(x)\right| & \leq \frac{3}{8} M_{4} h^{2}+\frac{8}{h^{2}}\|\tilde{Y}-Y\|_{\infty} .
\end{aligned}
$$


Proof. These results follow from the traditional cubic spline interpolation error theory $[1,3,7]$, Lemma 4 , and the following triangle inequality

$$
\begin{aligned}
\left|\widetilde{s}^{(k)}(x)-y^{(k)}(x)\right| \leq & \left|\widehat{s}^{(k)}(x)-s^{(k)}(x)\right| \\
& +\left|s^{(k)}(x)-y^{(k)}(x)\right|, \quad k=0,1,2 .
\end{aligned}
$$

4.2. Error Analysis for Method II. From (13) and Table 1, for $j=0,1, \ldots, n$, we have

$$
\begin{aligned}
& \tilde{\widetilde{s}}\left(x_{j}\right)=\frac{\tilde{y}_{j-1}+4 \tilde{y}_{j}+\tilde{y}_{j+1}}{6}, \\
& \widetilde{\widetilde{s}}^{\prime}\left(x_{j}\right)=\frac{\tilde{y}_{j+1}-\tilde{y}_{j-1}}{2 h}, \\
& \widetilde{\widetilde{s}}^{\prime \prime}\left(x_{j}\right)=\frac{\tilde{y}_{j-1}-2 \tilde{y}_{j}+\tilde{y}_{j+1}}{h^{2}} .
\end{aligned}
$$

It is a surprise to find that $\widetilde{\widetilde{s}}^{\prime}\left(x_{j}\right)$ and $\widetilde{\widetilde{s}}^{\prime \prime}\left(x_{j}\right)$ are the same as the well-known central numerical differentiation formulae.

Lemma 6. Let $\widetilde{\widetilde{s}}(x)$ be the noisy cubic spline quasi interpolant of $y(x)$ determined by (13). Then for $j=0,1, \ldots, n$, one has

$$
\begin{aligned}
\left|\widetilde{s}\left(x_{j}\right)-y\left(x_{j}\right)\right| & \leq \frac{1}{6} M_{2} h^{2}+\varepsilon, \\
\left|\widetilde{\widetilde{s}}^{\prime}\left(x_{j}\right)-y^{\prime}\left(x_{j}\right)\right| & \leq \frac{1}{6} M_{3} h^{2}+\frac{\varepsilon}{h}, \\
\left|\widetilde{\widetilde{s}}^{\prime \prime}\left(x_{j}\right)-y^{\prime \prime}\left(x_{j}\right)\right| & \leq \frac{1}{12} M_{4} h^{2}+\frac{4 \varepsilon}{h^{2}} .
\end{aligned}
$$

Proof. By (30), we have

$$
\begin{aligned}
& \left|\widetilde{\widetilde{s}}^{\prime \prime}\left(x_{j}\right)-y^{\prime \prime}\left(x_{j}\right)\right| \\
& =\left|\frac{\tilde{y}_{j-1}-2 \tilde{y}_{j}+\tilde{y}_{j+1}}{h^{2}}-y^{\prime \prime}\left(x_{j}\right)\right| \\
& =\left|\frac{y_{j-1}-2 y_{j}+y_{j+1}}{h^{2}}-y^{\prime \prime}\left(x_{j}\right)+\frac{\varepsilon_{j-1}-2 \varepsilon_{j}+\varepsilon_{j+1}}{h^{2}}\right| \\
& \leq \frac{1}{12} M_{4} h^{2}+\frac{4 \varepsilon}{h^{2}} \text {. }
\end{aligned}
$$

The proofs of (31) and (32) are similar, which are omitted.
Theorem 7. Let $\widetilde{\widetilde{s}}(x)$ be the noisy cubic spline quasi interpolant of $y(x)$ determined by (13). Then we have

$$
\begin{aligned}
|\widetilde{\widetilde{s}}(x)-y(x)| & \leq \frac{1}{384} M_{4} h^{4}+\frac{1}{24} M_{3} h^{3}+\frac{1}{6} M_{2} h^{2}+\frac{5}{4} \varepsilon \\
\left|\widetilde{\widetilde{s}}^{\prime}(x)-y^{\prime}(x)\right| & \leq \frac{5}{48} M_{4} h^{3}+\frac{1}{6} M_{3} h^{2}+\frac{3}{h} \varepsilon \\
\left|\widetilde{\widetilde{s}}^{\prime \prime}(x)-y^{\prime \prime}(x)\right| & \leq \frac{5}{24} M_{4} h^{2}+\frac{4}{h^{2}} \varepsilon
\end{aligned}
$$

Proof. We first prove (37). $\widetilde{\widetilde{s}}(x)$ is a cubic spline; hence $\widetilde{\widetilde{s}}^{\prime \prime}(x)$ is a piecewise continuous linear function over $[a, b]$ with respect to the partition $\Delta$. Let $L(x)$ be the piecewise linear interpolant to $y^{\prime \prime}(x)$ with respect to $\Delta$. For $j=1,2, \ldots, n$, let $\widetilde{\widetilde{s}}_{j}^{\prime \prime}(x)$ and $L_{j}(x)$ be the restriction of $\widetilde{\widetilde{s}}^{\prime \prime}(x)$ and $L(x)$ over $\left[x_{j-1}, x_{j}\right]$. Then we have

$$
\begin{aligned}
& \widetilde{\widetilde{s}}_{j}^{\prime \prime}(x)=\widetilde{\widetilde{s}}^{\prime \prime}\left(x_{j-1}\right) \frac{x_{j}-x}{h}+\widetilde{\widetilde{s}}^{\prime \prime}\left(x_{j}\right) \frac{x-x_{j-1}}{h}, \\
& L_{j}(x)=y^{\prime \prime}\left(x_{j-1}\right) \frac{x_{j}-x}{h}+y^{\prime \prime}\left(x_{j}\right) \frac{x-x_{j-1}}{h} .
\end{aligned}
$$

For $j=1,2, \ldots, n$, by (38) and (33), we get

$$
\begin{aligned}
\left|\widetilde{\widetilde{s}}_{j}^{\prime \prime}(x)-L_{j}(x)\right|= & \mid\left(\widetilde{\widetilde{s}}^{\prime \prime}\left(x_{j-1}\right)-y^{\prime \prime}\left(x_{j-1}\right)\right) \frac{x_{j}-x}{h} \\
& +\left(\widetilde{\widetilde{s}}^{\prime \prime}\left(x_{j}\right)-y^{\prime \prime}\left(x_{j}\right)\right) \frac{x-x_{j-1}}{h} \mid \\
\leq & \frac{1}{12} M_{4} h^{2}+\frac{4}{h^{2}} \varepsilon .
\end{aligned}
$$

Hence, for all $x \in[a, b]$, we have

$$
\left|\widetilde{\widetilde{s}}^{\prime \prime}(x)-L(x)\right| \leq \frac{1}{12} M_{4} h^{2}+\frac{4}{h^{2}} \varepsilon .
$$

Moreover, by the piecewise linear polynomial interpolation theory $[1,3,7]$, for all $x \in[a, b]$, we have

$$
\left|y^{\prime \prime}(x)-L(x)\right| \leq \frac{1}{8} M_{4} h^{2} .
$$

Then (37) follows immediately from (40) and (41).

Next, we prove (36). For $j=1,2, \ldots, n$, let $\widetilde{\widetilde{s}}_{j}^{\prime}(x)$ be the restriction of $\widetilde{\widetilde{s}}^{\prime}(x)$ over $\left[x_{j-1}, x_{j}\right]$. Then for $x \in\left[x_{j-1}, x_{j}\right]$, by (32) and (37), we have

$$
\left|\widetilde{\widetilde{s}}_{j}^{\prime}(x)-y^{\prime}(x)\right|= \begin{cases}\left|\int_{x_{j-1}}^{x}\left(\widetilde{\widetilde{s}}_{j}^{\prime \prime}(t)-y^{\prime \prime}(t)\right) d t+\left(\widetilde{\widetilde{s}}_{j}^{\prime}\left(x_{j-1}\right)-y^{\prime}\left(x_{j-1}\right)\right)\right|, & x \in\left[x_{j-1}, x_{j-1}+\frac{h}{2}\right] \\ \left|\int_{x_{j}}^{x}\left(\widetilde{\widetilde{s}}_{j}^{\prime \prime}(t)-y^{\prime \prime}(t)\right) d t+\left(\widetilde{\widetilde{s}}_{j}^{\prime}\left(x_{j}\right)-y^{\prime}\left(x_{j}\right)\right)\right|, & x \in\left[x_{j-1}+\frac{h}{2}, x_{j}\right]\end{cases}
$$




$$
\begin{aligned}
& \leq \begin{cases}\int_{x_{j-1}}^{x_{j-1}+h / 2}\left|\widetilde{\widetilde{s}}_{j}^{\prime \prime}(t)-y^{\prime \prime}(t)\right| d t+\left|\widetilde{\widetilde{s}}_{j}^{\prime}\left(x_{j-1}\right)-y^{\prime}\left(x_{j-1}\right)\right|, & x \in\left[x_{j-1}, x_{j-1}+\frac{h}{2}\right] \\
\int_{x_{j-1}+h / 2}^{x_{j}}\left|\widetilde{\widetilde{s}}_{j}^{\prime \prime}(t)-y^{\prime \prime}(t)\right| d t+\left|\widetilde{\widetilde{s}}_{j}^{\prime}\left(x_{j}\right)-y^{\prime}\left(x_{j}\right)\right|, & x \in\left[x_{j-1}+\frac{h}{2}, x_{j}\right]\end{cases} \\
& \leq \frac{5}{48} M_{4} h^{3}+\frac{1}{6} M_{3} h^{2}+\frac{3}{h} \varepsilon .
\end{aligned}
$$

Finally, we prove (35). For every subinterval $\left[x_{j-1}, x_{j}\right], j=$ $1,2, \ldots, n$, we give

$$
\begin{aligned}
& \alpha_{j 1}(x)=\frac{2\left(x-x_{j-1}\right)^{3}-3 h\left(x-x_{j-1}\right)^{2}+h^{3}}{h^{3}}, \\
& \alpha_{j 2}(x)=\frac{-2\left(x-x_{j-1}\right)^{3}+3 h\left(x-x_{j-1}\right)^{2}}{h^{3}}, \\
& \beta_{j 1}(x)=\frac{\left(x-x_{j}\right)^{2}\left(x-x_{j-1}\right)}{h^{2}}, \\
& \beta_{j 2}(x)=\frac{\left(x-x_{j}\right)\left(x-x_{j-1}\right)^{2}}{h^{2}} .
\end{aligned}
$$

They are very useful in cubic Hermite interpolation, and we also have

$$
\begin{gathered}
\alpha_{j 1}(x) \geq 0, \quad \alpha_{j 2}(x) \geq 0, \\
\beta_{j 1}(x) \geq 0, \quad \beta_{j 2}(x) \leq 0, \\
\alpha_{j 1}(x)+\alpha_{j 2}(x)=1, \\
\beta_{j 1}(x)-\beta_{j 2}(x)=\frac{\left(x_{j-1}-x\right)\left(x-x_{j}\right)}{h} \leq \frac{h}{4} .
\end{gathered}
$$

Let $\widetilde{\widetilde{s}}_{j}(x)=\left.\tilde{\widetilde{s}}(x)\right|_{\left[x_{j-1}, x_{j}\right]}$ be the restriction of $\widetilde{\widetilde{s}}(x)$ over $\left[x_{j-1}, x_{j}\right]$; then it can also be written as

$$
\begin{aligned}
\widetilde{\widetilde{s}}_{j}(x)= & \widetilde{\widetilde{s}}\left(x_{j-1}\right) \alpha_{j 1}(x)+\widetilde{\widetilde{s}}\left(x_{j}\right) \alpha_{j 2}(x) \\
& +\widetilde{\widetilde{s}}^{\prime}\left(x_{j-1}\right) \beta_{j 1}(x)+\widetilde{\widetilde{s}}^{\prime}\left(x_{j}\right) \beta_{j 2}(x) .
\end{aligned}
$$

Let

$$
\begin{aligned}
H_{j}(x)= & y\left(x_{j-1}\right) \alpha_{j 1}(x)+y\left(x_{j}\right) \alpha_{j 2}(x) \\
& +y^{\prime}\left(x_{j-1}\right) \beta_{j 1}(x)+y^{\prime}\left(x_{j}\right) \beta_{j 2}(x)
\end{aligned}
$$

be the cubic Hermite interpolant of $y(x)$ over $\left[x_{j-1}, x_{j}\right]$; then for $x \in\left[x_{j-1}, x_{j}\right]$, by using (31), (32), (44), (45), and (46), we have

$$
\begin{gathered}
\left|y(x)-H_{j}(x)\right| \leq \frac{1}{384} M_{4} h^{4}, \\
\left|\widetilde{\widetilde{s}}_{j}(x)-H_{j}(x)\right| \\
=\mid\left(\widetilde{\widetilde{s}}\left(x_{j-1}\right)-y\left(x_{j-1}\right)\right) \alpha_{j 1}(x) \\
+\left(\widetilde{\widetilde{s}}\left(x_{j}\right)-y\left(x_{j}\right)\right) \alpha_{j 2}(x) \\
\quad+\left(\widetilde{\widetilde{s}}^{\prime}\left(x_{j-1}\right)-y^{\prime}\left(x_{j-1}\right)\right) \beta_{j 1}(x) \\
\quad+\left(\widetilde{\widetilde{s}}^{\prime}\left(x_{j}\right)-y^{\prime}\left(x_{j}\right)\right) \beta_{j 2}(x) \mid \\
\leq\left(\frac{1}{6} M_{2} h^{2}+\varepsilon\right)+\frac{h}{4}\left(\frac{1}{6} M_{3} h^{2}+\frac{\varepsilon}{h}\right) \\
=\frac{1}{24} M_{3} h^{3}+\frac{1}{6} M_{2} h^{2}+\frac{5}{4} \varepsilon .
\end{gathered}
$$

By the triangle inequality, we get (35).

\section{Numerical Tests and Discussions}

5.1. Numerical Tests. In this section, we perform numerical tests by Matlab. The following examples

$$
\begin{aligned}
& f_{1}(x)=\frac{5}{1+x^{2}}, \quad x \in[-1,1], \\
& f_{2}(x)=e^{x}, \quad x \in[-1,1],
\end{aligned}
$$

are considered.

In every numerical test, the mesh size $h$ and the measurement noise bound $\varepsilon$ are both given. Because the measurement noises $\varepsilon_{i}(i=0,1, \ldots, n)$ are random, we let $\varepsilon_{i}=\varepsilon \cdot r_{i}$, where $r_{i}(i=0,1, \ldots, n)$ are random numbers and satisfy $-1 \leq r_{i} \leq 1$.

In Tables 5 and 6, Methods I-1, I-2, and I-3 represent Method I with two-point, three-point, and five-point approximate boundary derivatives, respectively. CSM represents the cubic spline method in [11]. $E_{0}, E_{1}$, and $E_{2}$ are the maximum absolute error of the function, the first order derivative, and the second order derivative, respectively. 
TABLE 5: Numerical results of $f_{1}(x)$.

\begin{tabular}{|c|c|c|c|c|c|c|c|}
\hline & & Method & $\mathrm{I}-1$ & $\mathrm{I}-2$ & $\mathrm{I}-3$ & II & CSM [11] \\
\hline \multirow{3}{*}{$\varepsilon=10^{-4}$} & \multirow{3}{*}{$h=0.2$} & $E_{0}$ & $7.8 \times 10^{-3}$ & $1.6 \times 10^{-3}$ & $5.9 \times 10^{-4}$ & $6.0 \times 10^{-2}$ & $4.4 \times 10^{-3}$ \\
\hline & & $E_{1}$ & $2.4 \times 10^{-1}$ & $4.7 \times 10^{-2}$ & $1.3 \times 10^{-2}$ & $1.4 \times 10^{-1}$ & $2.1 \times 10^{-2}$ \\
\hline & & $E_{2}$ & 4.16 & 0.75 & 0.41 & 0.38 & 0.45 \\
\hline \multirow{3}{*}{$\varepsilon=10^{-4}$} & \multirow{3}{*}{$h=0.1$} & $E_{0}$ & $2.0 \times 10^{-3}$ & $1.3 \times 10^{-4}$ & $1.2 \times 10^{-4}$ & $1.6 \times 10^{-2}$ & $3.4 \times 10^{-4}$ \\
\hline & & $E_{1}$ & $1.2 \times 10^{-1}$ & $4.7 \times 10^{-3}$ & $3.0 \times 10^{-3}$ & $3.8 \times 10^{-2}$ & $3.1 \times 10^{-3}$ \\
\hline & & $E_{2}$ & 4.29 & 0.15 & 0.14 & 0.10 & 0.15 \\
\hline \multirow{3}{*}{$\varepsilon=10^{-3}$} & \multirow{3}{*}{$h=0.2$} & $E_{0}$ & $8.0 \times 10^{-3}$ & $1.8 \times 10^{-3}$ & $1.3 \times 10^{-3}$ & $6.0 \times 10^{-2}$ & $4.5 \times 10^{-3}$ \\
\hline & & $E_{1}$ & $2.4 \times 10^{-1}$ & $4.7 \times 10^{-2}$ & $1.8 \times 10^{-2}$ & $1.5 \times 10^{-1}$ & $2.3 \times 10^{-2}$ \\
\hline & & $E_{2}$ & 4.17 & 0.75 & 0.43 & 0.39 & 0.45 \\
\hline \multirow{3}{*}{$\varepsilon=10^{-3}$} & \multirow{3}{*}{$h=0.1$} & $E_{0}$ & $2.2 \times 10^{-3}$ & $1.0 \times 10^{-3}$ & $9.1 \times 10^{-4}$ & $1.6 \times 10^{-2}$ & $9.4 \times 10^{-4}$ \\
\hline & & $E_{1}$ & $1.2 \times 10^{-1}$ & $1.6 \times 10^{-2}$ & $1.0 \times 10^{-2}$ & $4.0 \times 10^{-2}$ & $1.1 \times 10^{-2}$ \\
\hline & & $E_{2}$ & 4.29 & 0.65 & 0.42 & 0.29 & 0.44 \\
\hline
\end{tabular}

TABLE 6: Numerical results of $f_{2}(x)$.

\begin{tabular}{|c|c|c|c|c|c|c|c|}
\hline & & Method & I-1 & $\mathrm{I}-2$ & $\mathrm{I}-3$ & II & CSM [11] \\
\hline \multirow{3}{*}{$\varepsilon=10^{-5}$} & \multirow{3}{*}{$h=0.2$} & $E_{0}$ & $8.0 \times 10^{-3}$ & $9.8 \times 10^{-4}$ & $1.7 \times 10^{-5}$ & $1.3 \times 10^{-2}$ & $8.2 \times 10^{-5}$ \\
\hline & & $E_{1}$ & $6.8 \times 10^{-2}$ & $8.3 \times 10^{-3}$ & $2.3 \times 10^{-4}$ & $1.4 \times 10^{-2}$ & $1.1 \times 10^{-4}$ \\
\hline & & $E_{2}$ & 1.17 & 0.13 & $6.8 \times 10^{-3}$ & $7.3 \times 10^{-3}$ & $7.3 \times 10^{-3}$ \\
\hline \multirow{3}{*}{$\varepsilon=10^{-5}$} & \multirow{3}{*}{$h=0.1$} & $E_{0}$ & $2.0 \times 10^{-3}$ & $1.3 \times 10^{-4}$ & $1.0 \times 10^{-5}$ & $3.9 \times 10^{-3}$ & $1.2 \times 10^{-5}$ \\
\hline & & $E_{1}$ & $3.5 \times 10^{-2}$ & $2.2 \times 10^{-3}$ & $2.7 \times 10^{-4}$ & $4.1 \times 10^{-3}$ & $1.1 \times 10^{-4}$ \\
\hline & & $E_{2}$ & 1.21 & $7.5 \times 10^{-2}$ & $8.3 \times 10^{-3}$ & $3.6 \times 10^{-3}$ & $8.4 \times 10^{-3}$ \\
\hline \multirow{3}{*}{$\varepsilon=10^{-4}$} & \multirow{3}{*}{$h=0.2$} & $E_{0}$ & $8.0 \times 10^{-3}$ & $9.8 \times 10^{-4}$ & $1.0 \times 10^{-4}$ & $1.3 \times 10^{-2}$ & $1.2 \times 10^{-4}$ \\
\hline & & $E_{1}$ & $6.8 \times 10^{-2}$ & $8.3 \times 10^{-3}$ & $1.4 \times 10^{-3}$ & $1.4 \times 10^{-2}$ & $4.8 \times 10^{-4}$ \\
\hline & & $E_{2}$ & 1.17 & 0.13 & 0.019 & 0.010 & 0.021 \\
\hline \multirow{3}{*}{$\varepsilon=10^{-4}$} & \multirow{3}{*}{$h=0.1$} & $E_{0}$ & $2.0 \times 10^{-3}$ & $1.5 \times 10^{-4}$ & $7.3 \times 10^{-5}$ & $3.9 \times 10^{-3}$ & $8.5 \times 10^{-5}$ \\
\hline & & $E_{1}$ & $3.5 \times 10^{-2}$ & $2.6 \times 10^{-3}$ & $2.9 \times 10^{-3}$ & $4.0 \times 10^{-3}$ & $1.0 \times 10^{-3}$ \\
\hline & & $E_{2}$ & 1.22 & 0.08 & 0.08 & 0.03 & 0.09 \\
\hline
\end{tabular}

5.2. Discussions. Generally, the maximum absolute errors $E_{0}$, $E_{1}$, and $E_{2}$ vary if one of $\varepsilon$ and $h$ does. If $h$ is fixed and $\varepsilon$ decreases, then the maximum absolute errors $E_{0}, E_{1}$, and $E_{2}$ will decrease. But if $\varepsilon$ is fixed while $h$ decreases, the errors will not decrease necessarily; they maybe increase sometimes. See the theoretical results in Theorem 5 and Theorem 7 and the numerical results in Tables 5 and 6 .

When $h$ and $\varepsilon$ are both fixed in a specific test, it is easy to find that $E_{0}$ and $E_{1}$ of Method I-2 and Method I-3 are better than those of Method I-1 and Method II, while $E_{2}$ of Method I-3 and Method II are better than Method I-1 and Method I-2. See Tables 5 and 6.

It is very reasonable to compare our methods with the cubic spline method (CSM) in [11] because our methods are also based on cubic spline. From Tables 5 and 6, we find that the errors of Method I-3 are overall better than CSM in [11]. At the same time, $E_{2}$ of Method II are better than CSM in [11]. In summary, when approximating a function, we advise using Method I-3, Method I-2, and CSM [11]; when approximating its first order derivative, we advise using Method I-3 and CSM [11]; when approximating its second order derivative, we advise using Method I-3, Method II, and CSM [11].
When $\varepsilon=0$, Method I-1 and Method II are $O\left(h^{2}\right)$ methods, Method I-2 is an $O\left(h^{3}\right)$ method, and Method I3 is an $O\left(h^{4}\right)$ method. The cubic spline method (CSM) [11] is also an $O\left(h^{4}\right)$ method, while the method in [12] is an $O\left(h^{2}\right)$ method; the method in [13] is an $O\left(h^{2.5} \log h\right)$ method conditionally, only if the shape parameter $c=O(h)$ therein. Obviously, the approximation orders of Method I-2 and Method I-3 are higher than the methods in $[12,13]$, the approximation orders of Method I-1 and Method II equal that of the method in [12], and the approximation order of Method I-3 equals that of CSM [11]. Undoubtedly, our methods are full of approximation ability. Furthermore, [12, 13] have not studied first order and second order derivative approximations. At the same time, our methods are more suitable for noisy data than the methods in $[12,13]$. Hence, Method I-2, Method I-3, and CSM [11] are more preferable than others.

\section{Conclusions}

The explicit error bounds for a noisy lacunary cubic spline interpolation and a simple noisy cubic spline quasi interpolation are well studied in this paper; see Theorems 5 and 7. 
These new results are very useful in numerical approximation and related practical fields. Moreover, these results are also verified by some numerical examples. In a word, both theoretical analysis and numerical tests show that our methods are well behaved. We end the paper with the following remarks.

(i) The main contributions of the paper include (i) studying two new methods to approximate a function and its first order and second order derivatives from the given noisy data and (ii) analyzing the explicit error bounds for the methods.

(ii) The main advantages of our new methods include the following: (i) they are very simple; (ii) they are not only applicable to noisy data but also applicable to exact data; (iii) Method I-2 and Method I-3 have better performance in function approximation and first order derivative approximation than other methods; Method I-3 and Method II have better performance in second order derivative approximation than other methods.

\section{Conflict of Interests}

The authors declare that there is no conflict of interests regarding the publication of this paper.

\section{Acknowledgments}

The authors appreciate the reviewers and editors for their careful reading, valuable suggestions, and timely review and reply.

\section{References}

[1] E. Süli and D. F. Mayers, An Introduction to Numerical Analysis, Cambridge University Press, Cambridge, UK, 2003.

[2] C. A. Hall, "Optimal error bounds for cubic spline interpolation," Journal of Approximation Theory, vol. 16, no. 2, pp. 105$122,1976$.

[3] A. Quarteroni, R. Sacco, and F. Saleri, Numerical Mathematics, Springer, Berlin, Germany, 2nd edition, 2007.

[4] I. J. Schoenberg, "Contribution to the problem of approximation of equidistant data by analytic functions," Quarterly of Applied Mathematics, vol. 4, pp. 45-99 and 112-141, 1946.

[5] C. de Boor, A Practical Guide to Splines, Springer, New York, NY, USA, 1978.

[6] F. Lang and X. Xu, "A new cubic B-spline method for linear fifth order boundary value problems," Journal of Applied Mathematics and Computing, vol. 36, no. 1-2, pp. 101-116, 2011.

[7] R. H. Wang, Numerical Approximation, Higher Education Press, Beijing, China, 1999.

[8] L. L. Schumaker, Spline Functions: Basic Theory, Cambridge University Press, Cambridge, UK, 3rd edition, 2007.

[9] C. Zhu and W. Kang, "Numerical solution of Burgers-Fisher equation by cubic B-Spline quasi-interpolation," Applied Mathematics and Computation, vol. 216, no. 9, pp. 2679-2686, 2010.

[10] Z. W. Jiang and R. H. Wang, "An improved numerical solution of Burgers' equation by cubic B-spline quasi-interpolation," Journal of Information and Computational Science, vol. 7, no. 5, pp. 1013-1021, 2010.
[11] P. Sablonnière, "Univariate spline quasi-interpolants and applications to numerical analysis," Rendiconti del Seminario Matematico Università e Politecnico di Torino, vol. 63, no. 3, pp. 211-222, 2005.

[12] Z. M. Wu and R. Schaback, "Shape preserving properties and convergence of univariate multiquadric quasi-interpolation," Acta Mathematicae Applicatae Sinica, vol. 10, no. 4, pp. 441-446, 1994.

[13] L. Ling, "A univariate quasi-multiquadric interpolation with better smoothness," Computers \& Mathematics with Applications, vol. 48, no. 5-6, pp. 897-912, 2004. 


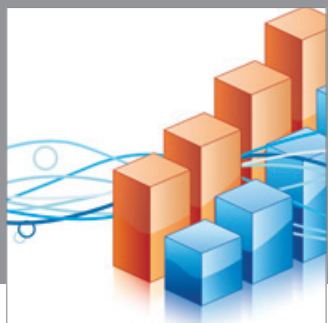

Advances in

Operations Research

mansans

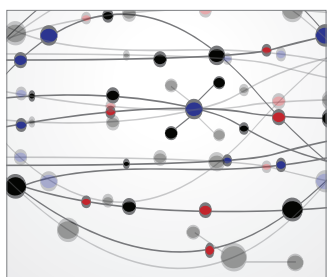

The Scientific World Journal
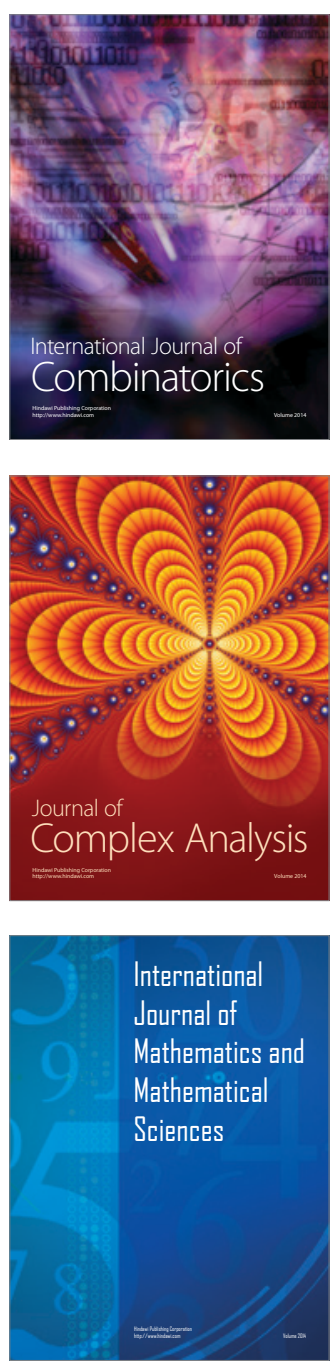
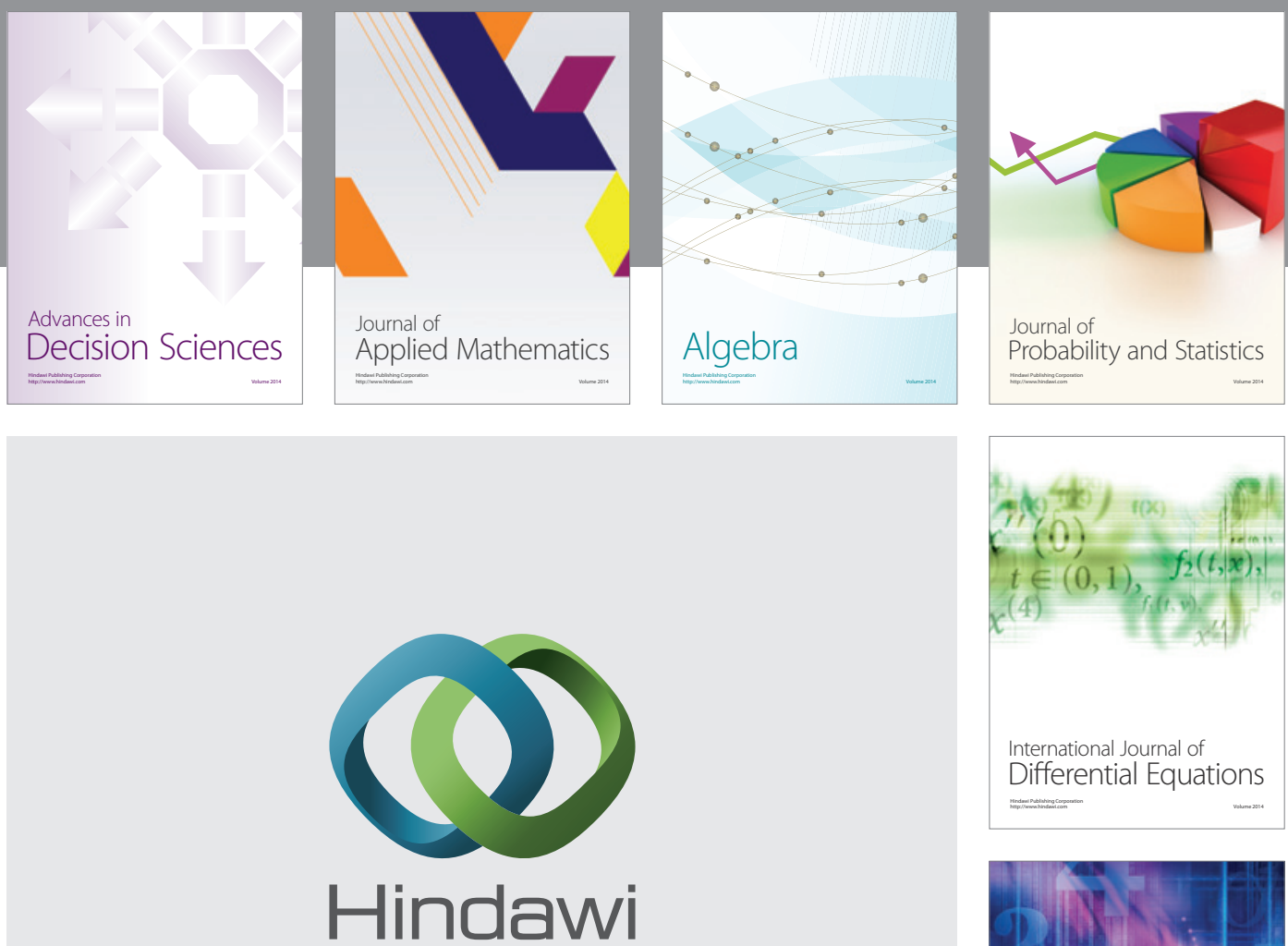

Submit your manuscripts at http://www.hindawi.com
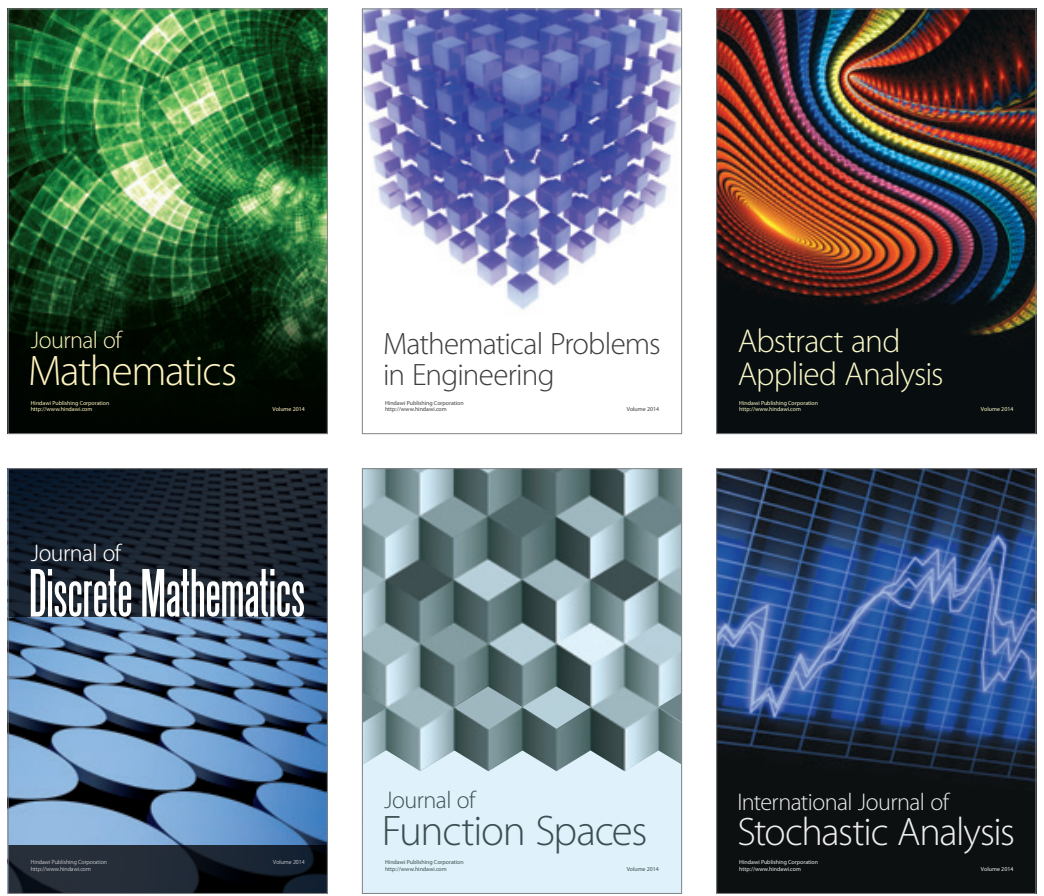

Journal of

Function Spaces

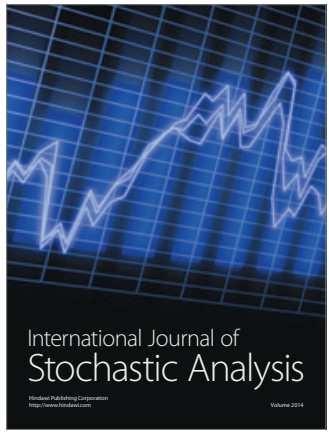

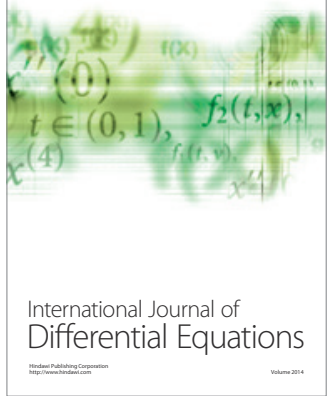
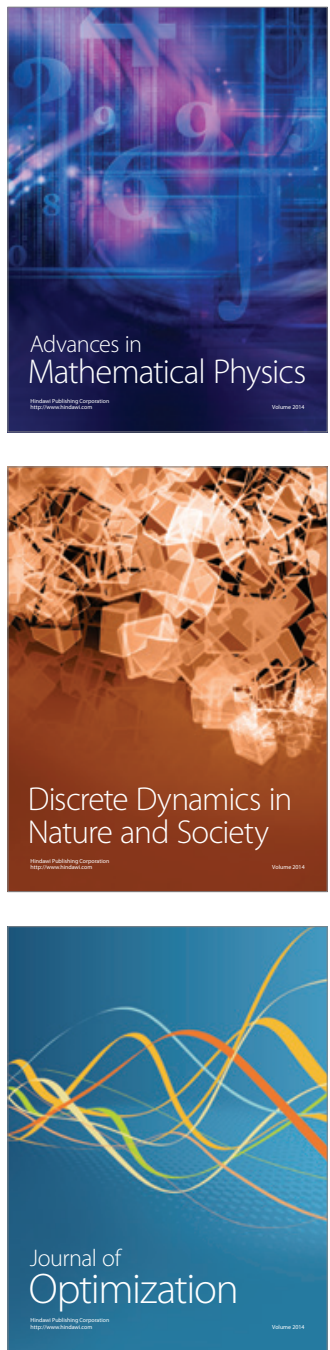